JOURNAL OF INTEGRAL EQUATIONS AND APPLICATIONS

Volume 3, Number 1, Winter 1991

\title{
A SLEWING BEAM PROBLEM
}

\author{
WOLFGANG DESCH, RICHARD K. MILLER, AND STEVEN B. SKAAR
}

\begin{abstract}
We consider feedback stabilization of a simple mechanical system involving a flexible viscoelastic beam. Though we use linear constitutive equations, nonlinearities come in by asymmetry of the geometric configuration of the system. The nonlinearities are handled as Lipschitz perturbations of a linear $C_{0}$-semigroup. Using Lyapunov techniques, we prove that solutions of the purely elastic stabilized system converge to zero weakly; if viscoelastic damping is present, the convergence is exponential in energy norm.
\end{abstract}

1. Introduction. In recent papers we have considered the model of a satellite set up in [8]. The system consists of a rigid hub with four identical flexible radial beams rigidly attached. The system is free to rotate and translate in a plane orthogonal to the axis of the hub. The beams are described by the Euler-Bernoulli model, based on a linearly viscoelastic constitutive equation for their material. A control torque can be applied on the hub in order to control its angular position. If we assume that all beams vibrate in phase, the model equations add up to a linear system of the type treated in $[\mathbf{3}]$ and $[\mathbf{4}]$. In these papers it is proved that linear feedback from angular position to torque can force the potential energy stored in deflection and the part of kinetic energy contained in rotation to decay to zero exponentially.

In the model described above the deformation of the flexible parts has no bearing on the location of the center of gravity, as the beams are arranged symmetrically around the axis, and their vibrations are assumed to be synchronized. The latter hypothesis is, of course, somewhat unrealistic. The former assumption imposes a severe restriction on the system's generality. We consider, therefore, the case of a mechanical system, where this symmetry is broken. The simplest one, and

Cooperation of R.M. and W.D. is supported by the NSF under grant DMS 8901574 and FWF within US Austrian Cooperative Science Program, P-6555. S. S. was supported by the U.S. Office of Naval Research under grant N00014-89-J-1533.

1980 Mathematics Subject Classification: 45K05, 93D15.

Keywords. Flexible structure, viscoelasticity, stabilization, Lyapunov techniques, nonlinear semigroups.

Copyright (C)1991 Rocky Mountain Mathematics Consortium 
the only one treated here, is obtained when we leave only one beam attached to the hub.

Below, we set up the equations for this system, based again on linear viscoelasticity. We will now encounter bilinear terms in the differential equations, which are due to the more complicated geometry of the object. (Notice that we do not consider the more complicated problem of handling nonlinear constitutive equations.) Fortunately, the nonlinearities come up as Lipschitz continuous perturbations of a semigroup similar to the kind treated in [4]. It is therefore easy to show well-posedness of the semilinear system. We can, however, no longer rely on spectral methods to prove stability. Instead, we utilize a Lyapunov function invariance princple for abstract dynamical systems developed in [2].

With a feedback from angular position and angular velocity to the control torque, even the purely elastic system can be stabilized in the sense that solutions tend to zero weakly. For a viscoelastic material, we require only feedback observing the angular position and get exponential stabilization in the sense of energy norm. This works for completely monotonic, exponentially decaying relaxation kernels, which may be singular or not at zero.

It is worth mentioning that the semilinear structure of the equations seems to be due to the simple mechanical system chosen. More complex systems, or even more accurate modelling of the system under consideration (e.g., curvature correction in the beam model), yield nonlinearities which occur in the boundary conditions or even in the coefficients of the partial differential equations, making the problem a quasilinear one. The quasilinear structure seems to be the generic one for such problems. However, it is much harder to treat. The present paper should, therefore, be regarded as a first attempt to treat nonlinearities caused by asymmetric geometry, rather than a general answer to this class of problems.

The paper is organized as follows. We derive the model equations from the Lagrangian principle in the next section. Section 3 settles the technicalities about well-posedness of the problem. Section 4 is then devoted to our central issue of proving stability by a Lyapunov function. 


\section{Derivation of the model equations.}

Equations for the elastic system. We consider a mechanical system consisting of a rigid hub of radius $R$ and a flexible appendage of length $L$ radially and rigidly attached to this hub. A control moment gyro is attached to the center of the hub, the torque $u(t)$ acting between the gyro and the hub at time $t$ will be controlled by a DC motor. The system is constrained to rotate (and translate) in a plane.

We describe the position of the system at time $t$ by the following coordinates:

$$
\begin{aligned}
& x(t), y(t) \text { position of the center of the hub, } \\
& \theta(t) \text { angular position of the hub, } \\
& \Phi(t) \text { angular position of the gyro, } \\
& w(t, z) \text { deflection from rest of the flexible rod } \\
& \quad \text { at distance } z \text { from the center of the hub. }
\end{aligned}
$$

The following physical constants will be required:

$R$ radius of the hub,

$L$ length of the flexible appendage,

$M$ mass of the hub plus the gyro,

$\rho$ mass per unit length of the appendage

$M_{T}$ total mass of the system: $\rho L+M$,

$I_{1}$ moment of inertia of the gyro,

$I_{2}$ moment of inertia of the hub,

$I_{T}$ total moment of inertia with undeflected appendage,

without gyro: $I_{2}+\left((R+L)^{3}-R^{3}\right) / 3 \rho$,

$I$ cross sectional moment of inertia of the appendage,

$E$ elastic modulus of the appendage.

The appendage will be modelled as a Euler-Bernoulli beam, which is linearly viscoelastic. For the derivation of the mechanical equations, however, we assume linear elasticity. Viscoelasticity will be introduced in the next subsection.

The potential energy of the system (by deflection of the rod) is

$$
E_{\mathrm{pot}}=\frac{1}{2} \int_{R}^{R+L} E I w_{z z}^{2} d z .
$$


The kinetic energy contains terms from the rotation of the gyro, the rotation of the hub, translation of the hub, and the motion of the appendage:

$$
\begin{aligned}
E_{\mathrm{kin}}= & \frac{1}{2} I_{1} \dot{\Phi}^{2}+\frac{1}{2} I_{2} \dot{\theta}^{2}+\frac{1}{2} M\left(\dot{x}^{2}+\dot{y}^{2}\right) \\
& +\frac{\rho}{2} \int_{R}^{R+L}\left[(-\dot{x} \sin \theta+\dot{y} \cos \theta+\dot{w}+z \dot{\theta})^{2}+(\dot{x} \cos \theta+\dot{y} \sin \theta)^{2}\right] d z,
\end{aligned}
$$

where dots denote partial derivatives with respect to time. The virtual work performed on the system by the control torque is

$$
\delta W=u(\delta \theta-\delta \Phi)
$$

The principle of minimal action states that, for any trajectory $\Phi, \theta, x, y, w$ in a time interval $[0, T]$ and any variation $\delta \Phi, \delta \theta, \delta x, \delta y, \delta w$ vanishing at 0 and $T$,

$$
\int_{0}^{T}\left[\delta E_{\mathrm{kin}}-\delta E_{\mathrm{pot}}+\delta W\right] d t=0
$$

This is

$$
\begin{aligned}
& 0=\int_{0}^{T}\left\{I_{1} \dot{\Phi} \delta \dot{\Phi}+I_{2} \dot{\theta} \delta \dot{\theta}+M(\dot{x} \delta \dot{x}+\dot{y} \delta \dot{y})\right. \\
& +\rho \int_{R}^{R+L}[(-\delta \dot{x} \sin \theta-\dot{x} \cos \theta \delta \theta+\delta \dot{y} \cos \theta-\dot{y} \sin \theta \delta \theta+\delta \dot{w}+z \delta \dot{\theta}) \\
& \quad(-\dot{x} \sin \theta+\dot{y} \cos \theta+\dot{w}+z \dot{\theta}) \\
& \quad+(\delta \dot{x} \cos \theta-\dot{x} \sin \theta \delta \theta+\delta \dot{y} \sin \theta+\dot{y} \cos \theta \delta \theta) \\
& \quad \cdot(\dot{x} \cos \theta+\dot{y} \sin \theta)] d z \\
& \left.\quad-\int_{R}^{R+L} w_{z z} \delta w_{z z} d z+u(\delta \theta-\delta \Phi)\right\} d t
\end{aligned}
$$


Thus

$$
\begin{aligned}
& 0=\int_{0}^{T}\left\{\delta \Phi\left[-I_{1} \ddot{\Phi}-u\right]\right. \\
& +\delta \theta\left[\rho \int_{R}^{R+L}[(-\dot{x} \cos \theta-\dot{y} \sin \theta)(-\dot{x} \sin \theta+\dot{y} \cos \theta+\dot{w}+z \dot{\theta})\right. \\
& +(-\dot{x} \sin \theta+\dot{y} \cos \theta)(\dot{x} \cos \theta+\dot{y} \sin \theta)] d z \\
& \left.-I_{2} \ddot{\theta}+u\right] \\
& +\delta x\left[-M \ddot{x}+\rho \int_{R}^{R+L} \frac{\partial}{\partial t}[\sin \theta(-\dot{x} \sin \theta+\dot{y} \cos \theta+\dot{w}+z \dot{\theta})\right. \\
& -\cos \theta(\dot{x} \cos \theta+\dot{y} \sin \theta)] d z] \\
& +\delta y\left[-M \ddot{y}+\rho \int_{R}^{R+L} \frac{\partial}{\partial t}[-\cos \theta(-\dot{x} \sin \theta+\dot{y} \cos \theta+\dot{w}+z \dot{\theta})\right. \\
& -\sin \theta(\dot{x} \cos \theta+\dot{y} \sin \theta)] d z] \\
& +\int_{R}^{R+L} \delta(w+z \theta)\left[\rho \frac{\partial}{\partial t}(\dot{x} \sin \theta-\dot{y} \cos \theta-\dot{w}-z \dot{\theta})\right. \\
& \left.-E I w_{z z z z}\right] d z \\
& \left.+\delta \theta E I \int_{R}^{R+L} z w_{z z z z} d z\right\} d t .
\end{aligned}
$$

Integrations by parts and rearrangements give

$$
\begin{aligned}
0=\int_{0}^{T}\left\{\delta \Phi\left[-I_{1} \ddot{\Phi}-u\right]\right. & \\
& +\delta \theta\left[-I_{2} \ddot{\theta}+u-p \rho \int_{R}^{R+L}(\dot{w}+z \dot{\theta}) d z-E I R w_{z z z}(R)\right. \\
& \left.+E I w_{z z}(R)\right]
\end{aligned}
$$




$$
\begin{aligned}
& +\delta x\left[-M_{T} \ddot{x}+\rho \dot{\theta} \cos \theta \int_{R}^{R+L}(\dot{w}+z \dot{\theta}) d z\right. \\
& \left.+\rho \sin \theta \int_{R}^{R+L}(\ddot{w}+z \ddot{\theta}) d z\right] \\
& +\delta y\left[-M_{T} \ddot{y}+\rho \dot{\theta} \sin \theta \int_{R}^{R+L}(\dot{w}+z \dot{\theta}) d z\right. \\
& \left.\quad-\rho \cos \theta \int_{R}^{R+L}(\ddot{w}+z \ddot{\theta}) d z\right] \\
& \left.+\int_{R}^{R+L} \delta(w+z \theta)\left[\rho(-\dot{q}-\ddot{w}-z \ddot{\theta})-E I w_{z z z z}\right] d z\right\} d t .
\end{aligned}
$$

Here

$$
p=\dot{x} \cos \theta+\dot{y} \sin \theta, \quad q=-\dot{x} \sin \theta+\dot{y} \cos \theta
$$

We also put

$$
W(t, z)=w(t, z)+z \theta(t) .
$$

We infer the following equations:

$$
\begin{aligned}
& I_{2} \ddot{\theta}+p \rho \int_{R}^{R+L} \dot{W} d z+\operatorname{EIRW}_{z z z}(R)-E I W_{z z}(R)=u, \\
& -M_{T} \ddot{x}+\rho \dot{\theta} \cos \theta \int_{R}^{R+L} \dot{W} d z+\rho \sin \theta \int_{R}^{R+L} \ddot{W} d z=0, \\
& -M_{T} \ddot{y}+\rho \dot{\theta} \sin \theta \int_{R}^{R+L} \dot{W} d z-\rho \cos \theta \int_{R}^{R+L} \ddot{W} d z=0,
\end{aligned}
$$

We observe that

$$
\begin{aligned}
-\sin \theta \ddot{x}+\cos \theta \ddot{y} & =\dot{q}+p \dot{\theta}, \\
\cos \theta \ddot{x}+\sin \theta \ddot{y} & =\dot{p}-q \dot{\theta} .
\end{aligned}
$$


Now, suitable linear combinations of (2.3) and (2.4) yield

$$
\begin{gathered}
M_{T}(\dot{q}+p \dot{\theta})+\rho \int_{R}^{R+L} \ddot{W} d z=0, \\
M_{T}(\dot{p}-q \dot{\theta})-\rho \dot{\theta} \int_{R}^{R+L} \dot{W} d z=0 .
\end{gathered}
$$

We insert (2.5) in (2.6) to obtain

$$
M \dot{q}+M_{T} p \dot{\theta}+E I W_{z z z}(R)=0 .
$$

Then (2.5) yields

$$
\ddot{W}=-\frac{E I}{\rho} W_{z z z z}+\frac{E I}{M} W_{z z z}(R)+\frac{M_{T}}{M} p \dot{\theta} .
$$

The fixed end boundary conditions in terms of $W$ are

$$
W(t, R)=R \theta, \quad W_{z}(t, R)=\theta .
$$

The free end boundary conditions are

$$
W_{z z}(t, R+L)=W_{z z z}(t, R+L)=0 .
$$

Let us finally remark that (2.1) is completely decoupled from the other equations. Hence, we may ignore this equation in the further course of our investigations.

Viscoelasticity. We assume now that the material of the flexible appendage is linearly viscoelastic, relating stress $\sigma$ and strain $\epsilon$ by the constitutive equation

$$
\sigma(t, z)=\int_{-\infty}^{t} a(t-s) \dot{\epsilon}(s, z) d s
$$

where $a$ is a completely monotonic function $a(t)=\int_{0}^{\infty} e^{-\lambda t} d g(\lambda)$ with some measure $d g$ on the positive half line. We assume that $a$ is integrable on compact subintervals of $[0, \infty)$, i.e., $\int_{0}^{\infty} 1 /(\lambda+\eta) d g(\lambda)<$ $\infty$ for all $\eta>0$, with $\lim _{t \rightarrow \infty} a(t)=E>0$, i.e., $d g$ has an atom $E$ at 0 . 
The elastic case is included in this setting since $d g$ may be the measure consisting of an atom $E$ at 0 . Furthermore, this model includes kernels that are (finite or countable) sums of exponentials as well as fractional derivative models.

The system $(2.2),(2.7),(2.8),(2.9)$ is now modified:

$I_{2} \ddot{\theta}+p \rho \int_{R}^{R+L} \dot{W} d z+I \int_{-\infty}^{t} a(t-s)\left[R \dot{W}_{z z z}(s, R)-\dot{W}_{z z}(s, R)\right] d s=u$,

(2.7) remains unchanged,

$$
M \dot{q}+M_{T} p \dot{\theta}+I \int_{-\infty}^{t} a(t-s) \dot{W}_{z z z}(s, R) d s=0
$$

$$
\ddot{W}=\int_{-\infty}^{t} a(t-s)\left[-\frac{I}{\rho} \dot{W}_{z z z z}(s, z)+\frac{I}{M} \dot{W}_{z z z}(s, R)\right] d s+\frac{M_{T}}{M} p \dot{\theta} .
$$

To rewrite the integrodifferential system as an ODE in a larger Hilbert space, we follow an approach in $[4]$. We define

$$
\phi(t, \lambda, z)=I \int_{-\infty}^{t} e^{-\lambda(t-s)} \dot{W}_{z z}(s, z) d s
$$

This yields

$$
\dot{\phi}(t, \lambda, z)=-\lambda \phi(t, \lambda, z)+I \dot{W}_{z z}(t, z) .
$$

Since

$$
I \int_{-\infty}^{t} a(t-s) \dot{W}_{z z}(s, z) d s=\int_{0}^{\infty} \phi(t, \lambda, z) d g(\lambda)
$$

we obtain

$$
\begin{gathered}
I_{2} \ddot{\theta}+p \rho \int_{R}^{R+L} \dot{W} d z+\int_{0}^{\infty}\left[R \phi_{z}(t, \lambda, R)-\phi(t, \lambda, R)\right] d g(\lambda)=u \\
M \dot{q}+M_{T} p \dot{\theta}+\int_{0}^{\infty} \phi_{z}(t, \lambda, R) d g(\lambda)=0
\end{gathered}
$$




$$
\ddot{W}=\int_{0}^{\infty}\left[-\frac{1}{\rho} \phi_{z z}(t, \lambda, z)+\frac{1}{M} \phi_{z}(t, \lambda, R)\right] d g(\lambda)+\frac{M_{T}}{M} p \dot{\theta}
$$

The free end boundary conditions can be rewritten in terms of $\phi$ :

$$
\int_{0}^{\infty} \phi(t, \lambda, R+L) d g(\lambda)=\int_{0}^{\infty} \phi_{z}(t, \lambda, R+L) d g(\lambda)=0 .
$$

The control motor. Let $v$ be the voltage driving the control motor. With inductivity $l$, resistance $r$, friction $f$, and motor constants $k_{1} \geq$ $k_{2}$, the relation between voltage, current $J$ and torque is

$$
\begin{gathered}
l \dot{J}=-r J+v-k_{1}(\dot{\theta}-\dot{\Phi}), \\
u=k_{2} J-f(\dot{\theta}-\dot{\Phi}) .
\end{gathered}
$$

We may ignore the inductivity of the coils, which is usually small, and put $l=0$. In order to stabilize the motion to an equilibrium at $\theta=0$, we feed back the voltage

$$
v=-\frac{r K_{1}}{k_{2}} \theta-\frac{r K_{2}}{k_{2}} \dot{\theta}+\left(k_{1}+\frac{f r}{k_{2}}\right)(\dot{\theta}-\dot{\Phi})
$$

with $K_{1}>0, K_{2} \geq 0$, so that we obtain a linear feedback law,

$$
u=-K_{1} \theta-K_{2} \dot{\theta}
$$

Summary of the equations. For convenience we summarize the system of equations we are going to investigate with their original equation numbers:

$$
\begin{gathered}
M_{T}(\dot{p}-q \dot{\theta})-\rho \dot{\theta} \int_{R}^{R+L} \dot{W} d z=0 . \\
\dot{\phi}(t, \lambda, z)=-\lambda \phi(t, \lambda, z)+I \dot{W}_{z z}(t, z) . \\
I_{2} \ddot{\theta}+p \rho \int_{R}^{R+L} \dot{W} d z+\int_{0}^{\infty}\left[R \phi_{z}(t, \lambda, R)-\phi(t, \lambda, R)\right] d g(\lambda)=u
\end{gathered}
$$




$$
\ddot{W}=\int_{0}^{\infty}\left[-\frac{1}{\rho} \phi_{z z}(t, \lambda, z)+\frac{1}{M} \phi_{z}(t, \lambda, R)\right] d g(\lambda)+\frac{M_{T}}{M} p \dot{\theta}
$$

$$
\int_{0}^{\infty} \phi(t, \lambda, R+L) d g(\lambda)=\int_{0}^{\infty} \phi_{z}(t, \lambda, R+L) d g(\lambda)=0 .
$$

$$
\dot{W}(t, R)=R \dot{\theta}, \quad \dot{W}_{z}(t, R)=\dot{\theta} .
$$

(The last equation is a differentiated version of (2.10).)

3. Well-posedness. In this section we put equations (2.7) to (2.20) in a functional analytic framework to prove existence and uniqueness of solutions. We exploit the fact that the nonlinearities in the model are Lipschitz continuous, and the linear part has the structure of a linear viscoelastic problem of the type treated in [4]. Therefore, the key to well-posedness is Lipschitz perturbation of a linear $C_{0}$-semigroup. For basic facts about semigroups we refer the reader to [5] or [7]. The concept of a weak solution has been adopted from [1] and [2].

We consider the vector

$$
\begin{aligned}
\mathbf{u}(t) & =(\theta(t), \dot{\theta}(t), p(t), q(t), \dot{W}(t, z) \phi(t, \lambda, z)) \\
& \in \mathbf{R}^{4} \times L^{2}([R, R+L], \mathbf{R}) \times L_{g}^{2}\left([0, \infty], L^{2}([R, R+L], \mathbf{R})\right)=X
\end{aligned}
$$


as state of the system. In vector form the equations read

$$
\begin{aligned}
& \frac{\partial}{\partial t}\left(\begin{array}{c}
\theta(t) \\
\dot{\theta}(t) \\
p(t) \\
q(t) \\
\dot{W}(t, z) \\
\phi(t, \lambda, z)
\end{array}\right)=\left(\begin{array}{c}
0 \\
-\frac{1}{I_{2}} \int_{0}^{\infty}\left[R \phi_{z}(t, \lambda, R)-\phi(t, \lambda, R)\right] d g(\lambda) \\
0 \\
-\frac{1}{M} \int_{0}^{\infty} \phi_{z}(t, \lambda, R) d g(\lambda) \\
\int_{0}^{\infty}\left[-\frac{1}{\rho} \phi_{z z}(t, \lambda, z)+\frac{1}{M} \phi_{z}(t, \lambda, R)\right] d g(\lambda) \\
-\lambda \phi(t, \lambda, z)+I \dot{W}_{z z}(t, z) \\
0 \\
\dot{\theta}(t) \\
+\left(\begin{array}{c}
-\frac{\rho}{K_{1}} \theta(t)-\frac{K_{2}}{I_{2}} \dot{\theta}(t) \\
-p(t) \\
0 \\
0 \\
0
\end{array}\right)+\left(\begin{array}{c}
R+L \\
I_{2} \\
-
\end{array}\right)+(t, z) d z \\
q(t) \dot{\theta}(t)+p(t)+\frac{\rho}{M_{T}} \int_{R}^{R+L} \dot{W}(t, z) d z \\
-\frac{M_{T}}{M} p(t) \dot{\theta}(t) \\
\frac{M_{T}}{M} p(t) \dot{\theta}(t) \\
0
\end{array}\right)
\end{aligned}
$$

with the appropriate boundary conditions. Abstractly, this is

$$
\dot{\mathbf{u}}(t)=\mathbf{A u}(t)+\mathbf{C u}(t)+\mathbf{F u}(t),
$$

with a linear unbounded operator $\mathbf{A}$, a linear bounded operator $\mathbf{C}$, and a Lipschitz continuous nonlinear operator $\mathbf{F}$. Before we explain these operators further, let us introduce an inner product in $\mathbf{X}$ by

$$
\begin{aligned}
& \left\langle\left(\begin{array}{c}
\theta_{1} \\
\dot{\theta}_{1} \\
p_{1} \\
q_{1} \\
\dot{W}_{1}(z) \\
\phi_{1}(\lambda, z)
\end{array}\right),\left(\begin{array}{c}
\theta_{2} \\
\dot{\theta}_{2} \\
p_{2} \\
q_{2} \\
\dot{W}_{2}(z) \\
\phi_{2}(\lambda, z)
\end{array}\right)\right\rangle \\
& =K_{1} \theta_{1} \theta_{2}+I_{2} \dot{\theta}_{1} \dot{\theta}_{2} \\
& +M_{T} p_{1} p_{2}+M q_{1} q_{2}+\rho \int_{R}^{R+L}\left(\dot{W}_{1}(z)+q_{1}\right)\left(\dot{W}_{2}(z)+q_{2}\right) d z \\
& \quad+\frac{1}{I} \int_{0}^{\infty} \int_{R}^{R+L} \phi_{1}(\lambda, z) \phi_{2}(\lambda, z) d z d g(\lambda) .
\end{aligned}
$$

(This inner product will also make up the Lyapunov function in Section 4.) 
The operator $\mathbf{A}$ is defined on the set $\operatorname{dom} \mathbf{A}$ of all

$$
(\theta, \dot{\theta}, p, q, \dot{W}(z), \phi(\lambda, z)) \in \mathbf{X}
$$

such that

$$
\begin{gathered}
\dot{W} \in W^{2,2}([R, R+L], \mathbf{R}), \quad \dot{W}(R)=R \dot{\theta}, \quad \dot{W}_{z}(R)=\dot{\theta} \\
I \dot{W}_{z z}(z)-\lambda \phi(\lambda, z) \in L_{g}^{2}\left([0, \infty], L^{2}([R, R+L], \mathbf{R})\right), \\
\int_{0}^{\infty} \phi(\lambda, z) d g(\lambda) \in W^{2,2}([R, R+L], \mathbf{R}), \\
\int_{0}^{\infty} \phi(\lambda, R+L) d g(\lambda)=\frac{\partial}{\partial z} \int_{0}^{\infty} \phi(\lambda, R+L) d g(\lambda)=0 .
\end{gathered}
$$

For such $(\theta, \dot{\theta}, p, q, \dot{W}(z), \phi(\lambda, z))$, we define

$$
\mathbf{A}\left(\begin{array}{c}
\theta \\
\dot{\theta} \\
p \\
q \\
\dot{W}(z) \\
\phi(\lambda, z)
\end{array}\right)=\left(\begin{array}{c}
0 \\
-\frac{1}{I_{2}}\left[\frac{\partial}{\partial z} \int_{0}^{\infty} R \phi(\lambda, R) d g(\lambda)-\int_{0}^{\infty} \phi(\lambda, R) d g(\lambda)\right] \\
0 \\
-\frac{1}{M} \frac{\partial}{\partial z} \int_{0}^{\infty} \phi(\lambda, R) d g(\lambda) \\
-\frac{1}{\rho} \frac{\partial^{2}}{\partial z^{2}} \int_{0}^{\infty} \phi(\lambda, z) d g(\lambda)+\frac{1}{M} \frac{\partial}{\partial z} \int_{0}^{\infty} \phi(\lambda, R) d g(\lambda) \\
-\lambda \phi(\lambda, z)+I \dot{W}_{z z}(z)
\end{array}\right) .
$$

Let us consider the state $\mathbf{u}=(\theta, \dot{\theta}, p, q, \dot{W}(z), \phi(\lambda, z))$ consisting of the four pieces

$$
\begin{gathered}
p \in \mathbf{R}, \\
\theta \in \mathbf{R}, \\
(\dot{\theta}, q, \dot{W}) \in \mathbf{R}^{2} \times L^{2}([R, R+L], \mathbf{R}), \\
\phi \in L_{g}^{2}\left([0, \infty], L^{2}([R, R+L], \mathbf{R})\right) .
\end{gathered}
$$

Then the first two components of $\mathbf{A u}$ are zero while the last two are

$$
\begin{aligned}
& -\mathbf{D} \int_{0}^{\infty} \phi d g(\lambda)=\left(\begin{array}{c}
-\frac{1}{I_{2}}\left[\frac{\partial}{\partial z} \int_{0}^{\infty} R \phi(\lambda, R) d g(\lambda)-\int_{0}^{\infty} \phi(\lambda, R) d g(\lambda)\right] \\
-\frac{1}{M} \frac{\partial}{\partial z} \phi(\lambda, R) d g(\lambda) \\
-\frac{1}{\rho} \frac{\partial^{2}}{\partial z^{2}} \int_{0}^{\infty} \phi(\lambda, z) d g(\lambda)+\frac{1}{M} \frac{\partial}{\partial z} \int_{0}^{\infty} \phi(\lambda, R) d g(\lambda)
\end{array}\right) \\
& -\lambda \phi+\mathbf{D}^{*}\left(\begin{array}{c}
\dot{\theta} \\
q \\
\dot{W}
\end{array}\right)=-\lambda \phi+I \dot{W}_{z z} .
\end{aligned}
$$


Here, $\mathbf{D}: L^{2}([R, R+L], \mathbf{R}) \supset \operatorname{dom} \mathbf{D} \rightarrow \mathbf{R}^{2} \times L^{2}([R, R+L], \mathbf{R})$ is defined by

$$
\begin{aligned}
& \operatorname{dom} \mathbf{D}=\left\{v \in W^{2,2}([R, R+L], \mathbf{R}): v(R+L)=v_{z}(R+L)=0\right\}, \\
& \mathbf{D} v=\left(\begin{array}{c}
\frac{1}{I_{2}}\left[R v_{z}(R)-v(R)\right] \\
\frac{1}{M} v_{z}(R) \\
\frac{1}{\rho} v_{z z}-\frac{1}{M} v_{z}(R)
\end{array}\right) . \\
& \mathbf{D}^{*}: \mathbf{R}^{2} \times L^{2}([R, R+L], \mathbf{R}) \supset \operatorname{dom} \mathbf{D}^{*} \rightarrow L^{2}([R, R+L], \mathbf{R}) \text { is defined } \\
& \text { by } \\
& \operatorname{dom} \mathbf{D}^{*}= \\
& \left\{\left(\begin{array}{c}
\dot{\theta} \\
q \\
\dot{W}
\end{array}\right) \in \mathbf{R}^{2} \times W^{2,2}([R, R+L], \mathbf{R}): \dot{W}(R)=R \dot{\theta}, \dot{W}_{z}(R)=\dot{\theta}\right\}, \\
& \mathbf{D}^{*}\left(\begin{array}{c}
\dot{\theta} \\
q \\
\dot{W}
\end{array}\right)=I \dot{W}_{z z}
\end{aligned}
$$

We take the inner products

$$
\left\langle v_{1}, v_{2}\right\rangle=\frac{1}{I} \int_{R}^{R+L} v_{1}(z) v_{2}(z) d z
$$

in $L^{2}([R, R+L], \mathbf{R})$ and

$$
\begin{aligned}
\left\langle\left(\begin{array}{c}
\dot{\theta}_{1} \\
q_{1} \\
\dot{W}_{1}
\end{array}\right),\left(\begin{array}{c}
\dot{\theta}_{2} \\
q_{2} \\
\dot{W}_{2}
\end{array}\right)\right\rangle=I_{2} \dot{\theta}_{1} \dot{\theta}_{2} & +M q_{1} q_{2} \\
& +\rho \int_{R}^{R+L}\left(\dot{W}_{1}(z)+q_{1}\right)\left(\dot{W}_{2}(z)+q_{2}\right) d z
\end{aligned}
$$

in $\mathbf{R}^{2} \times L^{2}([R, R+L], \mathbf{R})$. With these inner products, $\mathbf{D}$ and $\mathbf{D}^{*}$ are in fact adjoint to each other. This can be checked by an easy computation.

The operator $\mathbf{C}: \mathbf{X} \rightarrow \mathbf{X}$ is defined by

$$
\mathbf{C}\left(\begin{array}{c}
\theta \\
\dot{\theta} \\
p \\
q \\
\dot{W}(z) \\
\phi(\lambda, z)
\end{array}\right)=\left(\begin{array}{c}
\dot{\theta} \\
-\frac{K_{1}}{I_{2}} \theta-\frac{K_{2}}{I_{2}} \dot{\theta} \\
-p \\
0 \\
0 \\
0
\end{array}\right)
$$


Thus, $\mathbf{C}$ is a compact linear operator on $\mathbf{X}$.

When we ignore the $p$-component, which is completely uncoupled from the other components as far as $\mathbf{A}$ and $\mathbf{C}$ are concerned, we see that $\mathbf{A}+\mathbf{C}$ fits precisely in the structure of the operator $\mathcal{A}$ on page 404 of [4]. (The reader should not get confused by the fact that, in [4], the atom of $d g$ at 0 is treated separately, while we include it in the kernel $a$ here, so that the expressions with the constant $E$ in $[4]$ do not show up explicitly here.) We may, therefore, adopt the results of the paper quoted and obtain

Lemma 3.1. $\mathbf{A}+\mathbf{C}$ is the infinitesimal generator of a linear $C_{0^{-}}$ semigroup $\mathbf{S}_{0}$ on $\mathbf{X}$. Moreover, if $(a(\cdot)-E)$ decays exponentially, then $\mathbf{S}_{0}$ is exponentially stable.

Proof. [4] Theorem 2.3 states that $\mathbf{A}$ is a generator. Theorem 3.1 ibidem states that the essential growth rate of $\mathbf{S}_{0}$ is negative if $a-E$ decays exponentially. Using more of the methods in [4] and [3], we can rule out eigenvalues on the imaginary axis and therefore infer exponential stability.

We turn now to the nonlinear operator $\mathbf{F}: \mathbf{X} \rightarrow \mathbf{X}$ defined by

$$
\mathbf{F}\left(\begin{array}{c}
\theta \\
\dot{\theta} \\
p \\
q \\
\dot{W}(z) \\
\phi(\lambda, z)
\end{array}\right)=\left(\begin{array}{c}
0 \\
-\frac{\rho}{I_{2}} p \int_{R}^{R+L} \dot{W}(z) d z \\
q \dot{\theta}+p+\frac{\rho}{M_{T}} \int_{R}^{R+L} \dot{W}(z) d z \\
-\frac{M_{T}}{M} p \dot{\theta} \\
\frac{M_{T}}{M} p \dot{\theta} \\
0
\end{array}\right)
$$

We see that $\mathbf{F}$ depends, in fact, only on $p, \dot{\theta}$, and $\int_{R}^{R+L} \dot{W}(z) d z$, which can all be regarded as continuous linear functionals of the state. Thus $\mathbf{F}$ is continuous, even from the weak topology into the norm topology, so that the abstract system (3.2) fits in the framework of [2, Section 5, Subsection (b)]. In addition, $\mathbf{F}$ is continuously differentiable, in fact, infinitely often, the (Frechet) derivative being globally Lipschitz on bounded subsets of $\mathbf{X}$. 
By a weak solution of (3.2) we mean a function $\mathbf{u} \in C\left(\left[t_{0}, t_{1}\right), \mathbf{X}\right)$ satisfying the variation of parameters formula

$$
\mathbf{u}(t)=\mathbf{S}_{0}\left(t-t_{0}\right) \mathbf{u}\left(t_{0}\right)+\int_{t_{0}}^{t} \mathbf{S}_{0}(t-s) \mathbf{F u}(s) d s .
$$

This is consistent with the notion in $[\mathbf{2}]$.

LEMMA 3.2. For each $t_{0} \in \mathbf{R}$ and each initial value $\mathbf{u}\left(t_{0}\right)$ there exists a unique weak solution for (3.2) on a maximal interval of existence $\left[t_{0}, t_{\max }\right)$. Moreover, if $t_{\max }$ is finite, then $\mathbf{u}$ is unbounded on its interval of existence. If the initial value is contained in $\operatorname{dom} \mathbf{A}$, then $\mathbf{u} \in C^{1}\left(\left[t_{0}, t_{\max }\right), \mathbf{X}\right) \cap C\left(\left[t_{0}, t_{\max }\right), \operatorname{dom} \mathbf{A}\right)$; here, $\operatorname{dom} \mathbf{A}$ is equipped with the graph norm of $\mathbf{A}$. In this case, $\mathbf{u}$ satisfies the differential equation (3.2) for all t.

We remark that we will rule out the case that $\mathbf{u}$ is unbounded in the next section, so this Lemma, in fact, implies existence of a global solution.

PROOF. This is, in fact, a well known result about Lipschitz continuous perturbations of linear generators. The existence and uniqueness result can be obtained by using (3.3) as a fixed point equation for $\mathbf{u}$ in $C\left(\left[t_{0}, t_{1}\right], \mathbf{X}\right)$ for sufficiently small $t_{1}$, which has a unique solution by the Contraction Principle. A continuation argument works then as long as $\mathbf{u}$ remains bounded, so that the Lipschitz constant for $\mathbf{F}$ remains bounded. For $\mathbf{u}$ to stay in $\operatorname{dom} \mathbf{A}$ and to satisfy the differential equation, it is sufficient that the term $\mathbf{F u}$ entering the variation of parameters formula is in $C^{1}\left(\left[t_{0}, t_{\max }\right), \mathbf{X}\right)$. For this purpose we set up the same fixed point equation but check the contraction property with respect to $C^{1}\left(\left[t_{0}, t_{1}\right], \mathbf{X}\right)$. Again, $t_{1}$ has to be sufficiently small. If $\mathbf{u}, \mathbf{v}$ are in $C^{1}\left(\left[t_{0}, t_{1}\right], \mathbf{X}\right)$ with the same initial value and $\|\mathbf{u}(s)\| \leq M_{U}$, $\|\mathbf{v}(s)\| \leq M_{U}$ for some constant $M_{U},\|\dot{\mathbf{u}}(s)\| \leq M_{D},\|\dot{\mathbf{v}}(s)\| \leq M_{D}$ for 
another constant $M_{D}$, then

$$
\begin{aligned}
& \| \mathbf{S}_{0}\left(t-t_{0}\right) \mathbf{u}\left(t_{0}\right)+\int_{t_{0}}^{t} \mathbf{S}_{0}(t-s) \mathbf{F u}(s) d s \\
& -\mathbf{S}_{0}\left(t-t_{0}\right) \mathbf{u}\left(t_{0}\right)+\int_{t_{0}}^{t} \mathbf{S}_{0}(t-s) \mathbf{F v}(s) d s \| \\
& \quad \leq\left(t_{1}-t_{0}\right) M_{S} M_{F} \max _{s \in\left[t_{0}, t_{1}\right]}\|\mathbf{u}(s)-\mathbf{v}(s)\|,
\end{aligned}
$$

where $M_{S}=\max _{s \in\left[0, t_{1}-t_{0}\right]}\left\|\mathbf{S}_{0}(s)\right\|$ and $M_{F}$ is a Lipschitz constant for $\mathbf{F}$ valid for arguments bounded by $M_{U}$.

$$
\begin{aligned}
\| \frac{d}{d t}\left[\mathbf{S}_{0}\left(t-t_{0}\right) \mathbf{u}\left(t_{0}\right)+\int_{t_{0}}^{t} \mathbf{S}_{0}(t-s) \mathbf{F u}(s) d s\right] \\
-\frac{d}{d t}\left[\mathbf{S}_{0}\left(t-t_{0}\right) \mathbf{u}\left(t_{0}\right)+\int_{t_{0}}^{t} \mathbf{S}_{0}(t-s) \mathbf{F} \mathbf{v}(s) d s\right] \| \\
=\left\|\int_{t_{0}}^{t} \mathbf{S}_{0}(s) \frac{d}{d t}[\mathbf{F u}(t-s)-\mathbf{F} \mathbf{v}(t-s)] d s\right\| \\
=\left\|\int_{t_{0}}^{t} \mathbf{S}_{0}(s)[\nabla \mathbf{F}(\mathbf{u}(t-s)) \dot{\mathbf{u}}(t-s)-\nabla \mathbf{F}(\mathbf{v}(t-s)) \dot{\mathbf{v}}(t-s)] d s\right\| \\
\leq\left(t_{1}-t_{0}\right) M_{S} M_{D} M_{\nabla} \max _{s \in\left[t_{0}, t_{1}\right]}\|\mathbf{u}(s)-\mathbf{v}(s)\| \\
\quad+\left(t_{1}-t_{0}\right) M_{S} M_{U} M_{B} \max _{s \in\left[t_{0}, t_{1}\right]}\|\dot{\mathbf{u}}(s)-\dot{\mathbf{v}}(s)\|
\end{aligned}
$$

where $M_{\nabla}$ is a Lipschitz constant and $M_{B}$ is a bound for $\nabla \mathbf{F}$ for arguments bounded by $M_{U}$. From these equations it is easily seen that, for any $M_{U}, M_{D}$, we can find sufficiently small $t_{1}-t_{0}$ so that (3.3) is a contraction in $\left\{\mathbf{u} \in C^{1}\left(\left[t_{0}, t_{1}\right], \mathbf{X}\right):\|\mathbf{u}\| \leq M_{U},\|\dot{\mathbf{u}}\| \leq M_{D}\right\}$. We can therefore construct a solution in $C^{1}$, at least locally. The usual continuation argument yields that the solution stays in $C^{1}$ as long as $\|\mathbf{u}(t)\|$ and $\|\dot{\mathbf{u}}(t)\|$ stay bounded. We prove that $\|\dot{\mathbf{u}}\|$ cannot go to $\infty$ on any interval $\left[t_{0}, t_{1}\right)$, where the solution exists and is uniformly bounded, and $\dot{\mathbf{u}}$ exists. On such intervals we have bounds $M_{U}$ for $\|\mathbf{u}\|, M_{B}$ for 
$\|\nabla \mathbf{F}(\mathbf{u}(t))\|, M_{\omega} e^{\omega t}$ for $\left\|\mathbf{S}_{0}(t)\right\|$. Thus, by (3.3),

$$
\begin{aligned}
\|\dot{\mathbf{u}}(t)\|= & \left\|\frac{d}{d t}\left[\mathbf{S}_{0}\left(t-t_{0}\right) \mathbf{u}\left(t_{0}\right)+\int_{t_{0}}^{t} \mathbf{S}_{0}(t-s) \mathbf{F u}(s) d s\right]\right\| \\
= & \| \mathbf{A S}_{0}\left(t-t_{0}\right) \mathbf{u}\left(t_{0}\right)+\mathbf{S}_{0}\left(t-t_{0}\right) \mathbf{F u}\left(t_{0}\right) \\
& \quad+\int_{0}^{t-t_{0}} \mathbf{S}_{0}(s) \nabla \mathbf{F}(\mathbf{u}(t-s)) \dot{\mathbf{u}}(t-s) d s \| \\
\leq & M_{\omega} e^{\omega\left(t-t_{0}\right)}\left\|\dot{\mathbf{u}}\left(t_{0}\right)\right\|+\int_{0}^{t-t_{0}} M_{\omega} e^{\omega s} M_{B}\|\dot{\mathbf{u}}(t-s)\| .
\end{aligned}
$$

A Gronwall inequality argument shows that $\|\dot{\mathbf{u}}(t)\|$ is also bounded on that interval.

The following lemma states conservation of momentum along solutions. It will be needed for technical reasons in the analysis of the asymptotic behavior.

LEMMA 3.3. The following two functions are constant along trajectories of (3.1):

$$
\begin{aligned}
& M_{T} p \cos \theta-M_{T} q \sin \theta-\rho \sin \theta \int_{R}^{R+L} \dot{W} d z, \\
& M_{T} p \sin \theta+M_{T} q \cos \theta+\rho \cos \theta \int_{R}^{R+L} \dot{W} d z .
\end{aligned}
$$

ProOF. By a simple density argument, we may assume that the trajectory starts in $\operatorname{dom} \mathbf{A}$ and satisfies the differential equations in 
a strict sense. We can therefore compute the derivative

$$
\begin{gathered}
\frac{\partial}{\partial t}\left[M_{T} p \cos \theta-M_{T} q \sin \theta-\rho \sin \theta \int_{R}^{R+L} \dot{W} d z\right] \\
=\cos \theta\left[M_{T} \dot{p}-M_{T} q \dot{\theta}-\rho \dot{\theta} \int_{R}^{R+L} \dot{W} d z\right] \\
+\sin \theta\left[-M_{T} p \dot{\theta}-M_{T} \dot{q}-\rho \int_{R}^{R+L} \ddot{W} d z\right] \\
=\frac{M_{T}}{M} \sin \theta\left[-M \dot{q}-M_{T} p \dot{\theta}+\rho L p \dot{\theta}\right. \\
\quad+\frac{M}{M_{T}} \int_{R}^{R+L} \int_{0}^{\infty} \phi_{z z}(t, \lambda, z) d g(\lambda) d z \\
\left.\quad-\frac{\rho L}{M_{T}} \int_{0}^{\infty} \phi_{z}(t, \lambda, R) d g(\lambda)-\rho L p \dot{\theta}\right] \\
=0 . \quad
\end{gathered}
$$

The derivative of the second term is computed the same way.

4. Lyapunov function analysis. In this section we state and prove our main result.

MAIN THEOREM. Suppose that either a is nonconstant (i.e., the material is viscoelastically damped) or $K_{2} \neq 0$ (i.e., damping is provided by feedback opposite to angular velocity). Then each trajectory of (3.1) converges weakly to a state with $\theta=0, \dot{\theta}=0, \dot{W}=0, \phi=0$. Moreover, in the weak limit, $W=0$.

With suitable kernels, we obtain strong convergence to the limiting state:

COROLLARY. If a is nonconstant and $a-E$ is exponentially decaying (so that the support of $d g$, except the atom at 0 , is contained in an interval $[\epsilon, \infty)$ with $\epsilon>0)$, then the trajectories of (3.1) converge to their weak limit even with respect to the norm. 
Proof of THE COROLlary . By Lemma 3.2 the linearized semigroup $\mathbf{S}_{0}$ converges exponenitally to the zero state, while, from weak convergence, we infer that the nonlinear perturbation $\mathbf{F}(t)$ converges to 0 . It is an easy exercise in semigroup theory to show that the solutions of the nonlinear system converge in norm.

The remainder of this section is devoted to the proof of the Main Theorem. As this proof is rather technical, we split it up into several lemmas, and begin with a short overview.

The key idea is to define a Lyapunov function $V$ (4.1), consisting of mostly energy terms. $V$ decays along trajectories of (3.1), which can be seen from its derivative $G$. We infer that solutions are bounded in norm, thus trajectories are weakly compact. An invariance principle from [2] states that points of the weak $\omega$-limit set are starting points for trajectories where $V$ is constant. (We refer also to [6] for general information on abstract dynamical systems with compact trajectories.) Some technicalities have to be solved for adapting this theorem to our situation, since $G$ is not defined and not continuous on the whole state space (Lemmas 4.1 to 4.4 ). Given the invariance principle, we proceed to show that $V$ cannot be constant unless $\theta=0, \dot{\theta}=0, \dot{W}=0, \phi=0$. This is fairly easy in the case of viscoelastic damping, since $G$ contains terms from the potential energy bound to decay in the material, and $G=0$ means no potential energy, thus no deflection (Lemma 4.5). In the purely elastic case, energy in the material will not decay, thus it will not appear in $G$. Feedback based on position alone would give rise to oscillatory modes. Feeding back velocity as well, we see that $G=0$ implies $\dot{\theta}=0$. Proceeding from there in a rather technical manner, we end up with the fact that no deflection is possible if the angular position remains constant (Lemmas 4.6, 4.7).

We are now ready for the details. We define the Lyapunov function

$$
\begin{aligned}
& V(\theta, \dot{\theta}, p, q, \dot{W}, \phi)=\frac{K_{1}}{2} \theta^{2}+\frac{I_{2}}{2} \dot{\theta}^{2}+\frac{M_{T}}{2} p^{2}+\frac{M}{2} q^{2} \\
& +\frac{\rho}{2} \int_{R}^{R+L}(\dot{W}(z)+q)^{2} d z+\frac{1}{2 I} \int_{R}^{R+L} \int_{0}^{\infty} \phi^{2}(\lambda, z) d g(\lambda) d z .
\end{aligned}
$$


Lemma 4.1. For $(\theta, \dot{\theta}, p, q, \dot{W}, \phi) \in \operatorname{dom} \mathbf{A}$,

$$
\begin{aligned}
G(\theta, \dot{\theta}, p, q, \dot{W}, \phi) & =-\frac{\partial}{\partial t} V(\theta, \dot{\theta}, p, q, \dot{W}, \phi) \\
& =K_{2} \dot{\theta}^{2}+\frac{1}{I} \int_{R}^{R+L} \int_{0}^{\infty} \lambda \phi^{2} d g(\lambda) d z
\end{aligned}
$$

Proof. For $(\theta, \dot{\theta}, p, q, \dot{W}, \phi) \in \operatorname{dom} \mathbf{A}$, we multiply (2.7) by $p,(2.15)$ by $\phi / I$ and integrate with respect to $\lambda$ and $z,(2.16)$ by $\dot{\theta},(2.17)$ by $q$ and a suitable linear combination of (2.18), and (2.17) by $\rho(\dot{W}+q)$, integrating with respect to $z$. Adding up these equations we obtain

$$
\begin{aligned}
0=[ & \left.M_{T} p \dot{p}-M_{T} p q \dot{\theta}-p \rho \dot{\theta} \int_{R}^{R+L} \dot{W} d z\right] \\
& +\left[\frac{1}{I} \int_{R}^{R+L} \int_{0}^{\infty} \phi \dot{\phi} d g(\lambda) d z+\frac{1}{I} \int_{R}^{R+L} \int_{0}^{\infty} \lambda \phi^{2} d g(\lambda) d z\right. \\
& \left.-\int_{R}^{R+L} \dot{W}_{z z} \int_{0}^{\infty} \phi d g(\lambda) d z\right] \\
& +\left[I_{2} \ddot{\theta} \dot{\theta}+p \rho \dot{\theta} \int_{R}^{R+L} \dot{W} d z+\dot{\theta} \int_{0}^{\infty}\left[R \phi_{z}(t, \lambda, R)-\phi(t, \lambda, R)\right] d g(\lambda)\right. \\
& +\left[M q \dot{q}+M_{T} p q \dot{\theta}+q \int_{0}^{\infty} \phi_{z}(t, \lambda, R) d g(\lambda)\right] \\
& +\left[\rho \int_{R}^{R+L}(\ddot{W}+\dot{q})(\dot{W}+q) d z+\int_{R}^{R+L}(\dot{W}+q) \int_{0}^{\infty} \phi_{z z} d g(\lambda) d z\right]
\end{aligned}
$$


i.e.,

$$
\begin{aligned}
0= & M_{T} p \dot{p}+M q \dot{q}+K_{1} \theta \dot{\theta}+I_{2} \ddot{\theta} \dot{\theta} \\
& +\frac{1}{I} \int_{R}^{R+L} \int_{0}^{\infty} \phi \dot{\phi} d g(\lambda) d z+\rho \int_{R}^{R+L}(\ddot{W}+\dot{q})(\dot{W}+q) d z \\
+ & K_{2} \dot{\theta}^{2}+\frac{1}{I} \int_{R}^{R+L} \int_{0}^{\infty} \lambda \phi^{2} d g(\lambda) d z \\
+ & {\left[\int_{R}^{R+L} \dot{W} \int_{0}^{\infty} \phi_{z z} d g(\lambda) d z-\int_{R}^{R+L} \dot{W}_{z z} \int_{0}^{\infty} \phi d g(\lambda) d z\right.} \\
& \left.+\dot{\theta} \int_{0}^{\infty}\left[R \phi_{z}(t, \lambda, R)-\phi(t, \lambda, R)\right] d g(\lambda)\right] \\
+ & {\left[q \int_{0}^{\infty} \phi_{z}(t, \lambda, R) d g(\lambda)+q \int_{R}^{R+L} \int_{0}^{\infty} \phi_{z z} d g(\lambda) d z\right] . }
\end{aligned}
$$

Integration by parts and exploiting the boundary conditions shows that the terms in square brackets disappear so that we obtain the desired result. $\square$

We want to apply the methods of $[\mathbf{2}$, p. 255]. $\quad V$ is evidently a continuously Frechet differentiable function, and its gradient maps bounded sets into bounded sets. $G$, however, is only defined for $(\theta, \dot{\theta}, p, q, \dot{W}, \phi) \in \operatorname{dom} \mathbf{A}$ and has no continuous extension to the whole state space. This is due to the integral term $\int_{R}^{R+L} \int_{0}^{\infty} \lambda \phi^{2} d g(\lambda) d z$. Therefore, we need a slight modification of the proof of $[\mathbf{2}$, Theorem 5.14].

LEMMA 4.2. Let $(\theta(t), \dot{\theta}(t), p(t), q(t), \dot{W}(t, z), \phi(t, \lambda, z))$ be a (weak) solution of (3.1). We put

$$
V(t)=V(\theta(t), \dot{\theta}(t), p(t), q(t), \dot{W}(t, z), \phi(t, \lambda, z)) .
$$

Then

$$
\int_{R}^{R+L} \int_{0}^{\infty} \lambda \phi^{2} d g(\lambda) d z<\infty, \text { for } t>0 \text { a.e. }
$$

so that $G(\theta(t), \dot{\theta}(t), p(t), q(t), \dot{W}(t, z), \phi(t, \lambda, z))$ is defined a.e. 
Moreover,

$$
V(0)-V(t)=\int_{0}^{t} G(\theta(s), \dot{\theta}(s), p(s), q(s), \dot{W}(s, z), \phi(s, \lambda, z)) d s .
$$

In particular, $V$ is bounded and decreasing along trajectories, and

$$
\lim _{t \rightarrow \infty}(V(t)-V(t+\tau))=0, \text { for all } \tau>0 .
$$

Proof. If $(\theta(0), \dot{\theta}(0), p(0), q(0), \dot{W}(0, z), \phi(0, \lambda, z)) \in \operatorname{dom} \mathbf{A}$, then the solution stays in dom $\mathbf{A}$ for all $t>0$ and Lemma 4.1 can be applied immediately. Define

$$
G_{n}(\theta, \dot{\theta}, p, q, \dot{W}, \phi)=K_{2} \dot{\theta}^{2}+\frac{1}{I} \int_{R}^{R+L} \int_{0}^{n} \lambda \phi^{2} d g(\lambda) d z .
$$

By the monotone convergence principle,

$$
G(\theta, \dot{\theta}, p, q, \dot{W}, \phi)=\lim _{n \rightarrow \infty} G_{n}(\theta, \dot{\theta}, p, q, \dot{W}, \phi) .
$$

Since dom $\mathbf{A}$ is dense and $V$ and $G_{n}$ are continuous on the whole state space, we have

$$
V(0)-V(t) \geq \int_{0}^{t} G_{n}(\theta(s), \dot{\theta}(s), p(s), q(s), \dot{W}(s, z), \phi(s, \lambda, z)) d s .
$$

This yields an upper bound for $G_{n}$ in the $L_{1}$-norm, and the desired result can be inferred by the principle of monotone convergence. $\square$

LEMMA 4.3. Let $\left(\theta_{n}(t), \dot{\theta}_{n}(t), p_{n}(t), q_{n}(t), \dot{W}_{n}(t, z), \phi_{n}(t, \lambda, z)\right)$ be a sequence of trajectories converging pointwise weakly to $(\theta(t), \dot{\theta}(t), p(t)$, $q(t), \dot{W}(t, z), \phi(t, \lambda, z)) . \quad$ Put $V_{n}(t)=V\left(\theta_{n}(t), \dot{\theta}_{n}(t), p_{n}(t), q_{n}(t)\right.$, $\left.\dot{W}_{n}(t, z), \phi_{n}(t, \lambda, z)\right)$.

Then

$$
\varliminf_{n \rightarrow \infty}\left(V_{n}(0)-V_{n}(t)\right) \geq V(0)-V(t)
$$


ProOF. We have to show

$$
\varliminf_{n \rightarrow \infty} \int_{0}^{t} G\left(\theta_{n}(s), \ldots, \phi_{n}(s, \lambda, z)\right) d s \geq \int_{0}^{t} G(\theta(s), \ldots, \phi(s, \lambda, z)) d s .
$$

Put

$$
\gamma_{n}=G\left(\theta_{n}(s), \dot{\theta}_{n}(s), p_{n}(s), q_{n}(s), \dot{W}_{n}(s, z), \phi_{n}(s, \lambda, z)\right),
$$

and

$$
\gamma=G(\theta(s), \dot{\theta}(s), p(s), q(s), \dot{W}(s, z), \phi(s, \lambda, z)) .
$$

By convexity of $G$ and weak convergence of the trajectories, one can easily show that, for $s \in[0, t]$ a.e.,

$$
\varliminf_{n \rightarrow \infty} \gamma_{n}(s) \geq \gamma(s)
$$

Let $\epsilon>0$ and $M_{n}=\left\{s \in[0, t]: \gamma_{m}(s) \leq \gamma(s)+\epsilon\right.$ for $\left.m \geq n\right\}$. Notice that the measure of $[0, t] \backslash M_{n}$ converges to 0 . Now

$$
\begin{aligned}
\int_{0}^{t} \gamma(s) d s & =\int_{[0, t] \backslash M_{n}} \gamma(s) d s+\int_{M_{n}} \gamma(s) d s \\
& \leq \int_{[0, t] \backslash M_{n}} \gamma(s) d s+\int_{M_{n}} \gamma_{n}(s) d s+t \epsilon \\
& \leq \int_{[0, t] \backslash M_{n}} \gamma(s) d s+\int_{0}^{t} \gamma_{n}(s) d s+t \epsilon \\
& \rightarrow 0+\underline{\lim _{n \rightarrow \infty}} \int_{0}^{t} \gamma_{n}(s) d s+t \epsilon
\end{aligned}
$$

for a suitable subsequence. As $\epsilon$ was arbitrarily small, this proves the claim. $\square$

We recall that a point $(\theta, \dot{\theta}, p, q, \dot{W}, \phi)$ is said to lie in the weak $\omega$-limit set of (3.1) iff there is some trajectory $(\theta(t), \dot{\theta}(t), p(t), q(t), \dot{W}(t), \phi(t))$ and a sequence $t_{n} \rightarrow \infty$ such that

$$
\left(\theta\left(t_{n}\right), \dot{\theta}\left(t_{n}\right), p\left(t_{n}\right), q\left(t_{n}\right), \dot{W}\left(t_{n}\right), \phi\left(t_{n}\right)\right) \rightarrow(\theta, \dot{\theta}, p, q, \dot{W}, \phi)
$$


in the weak topology.

LEMMA 4.4. Any $(\theta, \dot{\theta}, p, q, \dot{W}, \phi)$ in the weak $\omega$-limit set of (3.1) is the initial value for a weak solution with

$$
G(\theta(t), \dot{\theta}(t), p(t), q(t), \dot{W}(t, z), \phi(t, \lambda, z))=0
$$

ProOF. The lemmas above allow us to follow the lines of the proof of Theorem 5.14 in [2], so that we may apply Theorem 3.5 ibidem to see that $V$ is constant along the trajectory starting at $(\theta, \dot{\theta}, p, q, \dot{W}, \phi)$. We define $G_{n}$ as in the proof of Lemma 4.2 and infer that $G_{n}$ vanishes everywhere on this trajectory. The principle of monotone convergence, applied at each point of the trajectory, shows that $G$ is defined and equals zero everywhere.

We will prove that the only possible weak limit is zero. The boundedness of the trajectories implies then that each solution converges weakly to zero.

LEMMA 4.5. If $d g$ is not just an atom at 0 (i.e., the material is not elastic), then the only points in the weak $\omega$-limit set of (3.1) are of the form $(0,0, p, q, 0,0)$.

ProOf. Let $(\theta, \dot{\theta}, p, q, \dot{W}, \phi)$ be in the weak $\omega$-limit set of (3.1). From Lemma 4.4 we infer that $G=0$ along the trajectory $(\theta(t), \dot{\theta}(t), p(t), q(t)$, $\dot{W}(t), \phi(t))$ starting at $(\theta, \dot{\theta}, p, q, \dot{W}, \phi)$. In particular, $\phi(t, \lambda, z)=0$ for almost all $\lambda$ except 0 . From (2.15) we infer that $\dot{W}_{z z}(t, z)=0$. Together with the boundary conditions (2.21) this implies $\dot{W}(t, z)=z \dot{\theta}$. By (2.15) $\phi$ is independent of $t$. (2.18) now reads

$$
z \ddot{\theta}=-\frac{E}{\rho} \phi_{z z}(0, z)+\frac{E}{M} \phi_{z}(0, R)+\frac{M_{T}}{M} p \dot{\theta} .
$$

As $z \ddot{\theta}$ and $p \dot{\theta}$ are now the only possibly time dependent entries in this equation and the latter is independent of $z$, we infer that, in fact, both 
are independent of $t$. In particular, $\theta$ is a quadratic polynomial, but as $V$ is bounded along the trajectory, $\theta$ must be constant. We now have

$$
\theta_{z z}(0, z)=\frac{\rho}{M} \phi_{z}(0, R),
$$

so that (with boundary condition (2.19))

$$
0=\phi_{z}(0, R+L)=\left(1+\frac{\rho L}{M}\right) \phi_{z}(0, R)
$$

and, finally, $\phi_{z}$ vanishes everywhere. By the free end boundary condition, $\phi$ vanishes as well. The whole left-hand side of (2.16) has disappeared and we are left with $u=0$, thus $\theta=0$.

The purely elastic case is more complicated. We require the following lemma.

Lemma 4.6. For no $x \geq 0$ with $1+\cos x \cosh x \neq 0$ are the following two inequalities simultaneously satisfied:

$$
\begin{gathered}
\frac{\sin x \cosh x+\cos x \sinh x}{1+\cos x \cosh x}<0, \\
\frac{\sin x \sinh x}{1+\cos x \cosh x}>0 .
\end{gathered}
$$

PROOF. If $x=\pi(n+1 / 2)$ with $n=1,2, \ldots$, then (4.3) reduces to $(-1)^{n} \cosh x<0$, and (4.4) reduces to $(-1)^{n} \sinh x>0$. Since $\cosh x$ and $\sinh x$ are positive, this is impossible.

If $x \neq \pi(n+1 / 2)$, the pair of inequalities reduces to

$$
\begin{aligned}
& \frac{\cos x \cosh x}{1+\cos x \cosh x}(\tan x+\tanh x)<0, \\
& \frac{\cos x \cosh x}{1+\cos x \cosh x}(\tan x \tanh x)>0 .
\end{aligned}
$$


In order to satisfy both inequalities, $\tan x+\tanh x$ and $\tan x \tanh x$ must be of opposite sign. Thus

$$
0<-\tan x<\tanh x
$$

Now (4.3) and (4.4) hold simultaneously if and only if

$$
0>\cos x>-\frac{1}{\cosh x}
$$

Then

$$
\begin{aligned}
1-\frac{1}{\cosh ^{2} x} & =\tanh ^{2} x>\tan ^{2} x \\
& =\frac{1}{\cos ^{2} x}-1>\cosh ^{2} x-1 .
\end{aligned}
$$

Thus,

$$
\left(\cosh ^{2} x-1\right)^{2}=\cosh ^{4} x-2 \cosh ^{2} x+1<0,
$$

which is impossible.

LEMMA 4.7. Suppose that $d g$ consists only of the atom $E$ at 0 (i.e., $a(t)=E$ for all $t)$ and that $K_{2}>0$. Then the only points in the weak $\omega$-limit set of (3.1) are of the form $(0,0, p, q, 0,0)$.

Proof. Let $(\theta, \dot{\theta}, p, q, \dot{W}, \phi)$ be in the weak $\omega$-limit set of (3.1). Again Lemma 4.4 implies that $G=0$ along the trajectory $(\theta(t), \dot{\theta}(t), p(t), q(t)$, $\dot{W}(t), \phi(t))$ starting at $(\theta, \dot{\theta}, p, q, \dot{W}, \phi)$. Since $K_{2}>0$, we infer that $\dot{\theta}=0$, thus $\theta$ is constant. (3.1) reduces to

$$
\begin{gathered}
M_{T} \dot{p}=0, \\
\dot{\phi}(t, z)=I \dot{W}_{z z}(t, z), \\
p \rho \int_{R}^{R+L} \dot{W} d z+E\left[R \phi_{z}(t, R)-\phi(t, R)\right]=u, \\
M \dot{q}+E \phi_{z}(t, R)=0,
\end{gathered}
$$




$$
\begin{gathered}
\ddot{W}=E\left[-\frac{1}{\rho} \phi_{z z}(t, z)+\frac{1}{M} \phi_{z}(t, R)\right], \\
u=-K_{1} \theta, \\
\phi(t, R+L)=\phi_{z}(t, R+L)=0, \\
\dot{W}(t, R)=0, \quad \dot{W}_{z}(t, R)=0 .
\end{gathered}
$$

(We have suppressed the argument $\lambda$ in $\phi$, since $\phi$ is only defined at $\lambda=0$, as the measure $d g$ vanishes on $(0, \infty)$.) We infer immediately that $p(t)$ and $\theta(t)$ are constant.

Consider the operator $\mathbf{B}$ defined on

$$
\begin{gathered}
\operatorname{dom} \mathbf{B}=\left\{(W, \phi) \in W^{2,2}([R, R+L], \mathbf{R}) \times W^{2,2}([R, R+L], \mathbf{R}):\right. \\
\left.W(R)=W_{z}(R)=0, \phi(R+L)=\phi_{z}(R+L)=0\right\} \\
\subset L^{2}([R, R+L], \mathbf{R}) \times L^{2}([R, R+L], \mathbf{R})
\end{gathered}
$$

by

$$
\mathbf{B}(W, \phi)=\left(\frac{E}{\rho} \phi_{z z},-I W_{z z}\right) .
$$

$\mathbf{B}$ is skew-adjoint with respect to the inner product

$$
\left\langle\left(W_{1}, \phi_{1}\right),\left(W_{2}, \phi_{2}\right)\right\rangle=\rho \int_{R}^{R+L} W_{1} W_{2} d z+\frac{E}{I} \int_{R}^{R+L} \phi_{1} \phi_{2} d z
$$

on $L^{2}([R, R+L], \mathbf{R}) \times L^{2}([R, R+L], \mathbf{R})$. Let $\mathbf{e}=(1,0) \in L^{2}([R, R+$ $L], \mathbf{R}) \times L^{2}([R, R+L], \mathbf{R})$, where 1 denotes the constant function 1 . The equations (4.6) and (4.9), together with all boundary conditions, can be written in the form

$$
\begin{aligned}
\frac{\partial}{\partial t}(\dot{W}(t, \cdot), \phi(t, \cdot)) & =-\mathbf{B}(\dot{W}(t, \cdot), \phi(t, \cdot))-\frac{1}{M}\langle\mathbf{e}, \mathbf{B}(\dot{W}(t, \cdot), \phi(t, \cdot))\rangle \mathbf{e} \\
& =\mathbf{H B}(\dot{W}(t, \cdot), \phi(t, \cdot)),
\end{aligned}
$$

where $\mathbf{H}=\mathrm{id}+(1 / M)\langle\mathbf{e}, \cdot\rangle \mathbf{e}$ is evidently a positive definite, self-adjoint operator. Consequently, $\mathbf{H}^{-1}$ exists as a bounded, positive definite 
operator, and the operator $\mathbf{H B}$ is skew-adjoint with respect to the inner product $\left\langle\cdot, \mathbf{H}^{-1} \cdot\right\rangle$. It has, therefore, a spectral resolution, and solutions may be written in the form

$$
(\dot{W}(t, \cdot), \phi(t, \cdot))=\sum_{j=1}^{\infty} \cos \left(\nu_{j} t\right) \mathbf{u}_{j}+\sin \left(\nu_{j} t\right) \mathbf{v}_{j},
$$

$\mathbf{u}_{j}, \mathbf{v}_{j}$ being suitable vectors in $L^{2}([R, R+L], \mathbf{R}) \times L^{2}([R, R+L], \mathbf{R})$.

Notice that (4.7) takes the form

$$
\sum_{j=1}^{\infty} \cos \left(\nu_{j} t\right) u_{j}+\sin \left(\nu_{j} t\right) v_{j}=-K_{1} \theta
$$

where the coefficients $u_{j}, v_{j}$ contain all information coming from $\dot{W}$, $\phi_{z}$, and $\phi$. By linear independence of sums of exponentials we infer that all $u_{j}, v_{j}$ as well as $\theta$ are zero. Suppose that $\dot{W}$ or $\phi$ do not vanish. In this case there is some nonzero $\mathbf{u}_{j}$ or $\mathbf{v}_{j}$. Then the components of $\cos \left(\nu_{j} t\right) \mathbf{u}_{j}+\sin \left(\nu_{j} t\right) \mathbf{v}_{j}$ provide a nontrivial solution to the system (4.6), (4.9) with all boundary conditions, and since $u_{j}=v_{j}=0$, they also satisfy (4.7) with $u=0$.

Let $U$ and $V$ be the first components of $\mathbf{u}_{j}, \mathbf{v}_{j}$. By (4.6), the second components are $-\left(I / \nu_{j}\right) V_{z z},\left(I / \nu_{j}\right) U_{z z}$. Thus (4.9) yields

$$
\begin{aligned}
U(z) & =\frac{E I}{\rho \nu_{j}^{2}} U_{z z z z}(z)-\frac{E I}{M \nu_{j}^{2}} U_{z z z}(R), \\
V(z) & =\frac{E I}{\rho \nu_{j}^{2}} V_{z z z z}(z)-\frac{E I}{M \nu_{j}^{2}} V_{z z z}(R) .
\end{aligned}
$$

The boundary conditions are

$$
\begin{gathered}
U(R)=U_{z}(R)=U_{z z}(R+L)=U_{z z z}(R+L)=0 \\
V(R)=V_{z}(R)=V_{z z}(R+L)=V_{z z z}(R+L)=0 .
\end{gathered}
$$

(4.7) is rewritten

$$
\begin{gathered}
p \rho \nu_{j} \int_{R}^{R+L} U d z-\frac{E I R}{\nu_{j}} V_{z z z}(R)+\frac{E I}{\nu_{j}} V_{z z}(R)=0, \\
-p \rho \nu_{j} \int_{R}^{R+L} V d z-\frac{E I R}{\nu_{j}} U_{z z z}(R)+\frac{E I}{\nu_{j}} U_{z z}(R)=0 .
\end{gathered}
$$


Using (4.13) and the boundary conditions, we can rewrite the last equation as

$$
\begin{aligned}
& -\frac{p E I}{\nu_{j}} U_{z z z}(R)-\frac{p \rho L E I}{\nu_{j} M} U_{z z z}(R)-\frac{E I R}{\nu_{j}} V_{z z z}(R)+\frac{E I}{\nu_{j}} V_{z z}(R)=0, \\
& -\frac{p E I}{\nu_{j}} V_{z z z}(R)-\frac{p \rho L E I}{\nu_{j} M} V_{z z z}(R)+\frac{E I R}{\nu_{j}} U_{z z z}(R)-\frac{E I}{\nu_{j}} U_{z z}(R)=0,
\end{aligned}
$$

i.e.,

$$
\begin{aligned}
& -\frac{M_{T} p}{M} U_{z z z}(R)-R V_{z z z}(R)+V_{z z}(R)=0, \\
& \frac{M_{T} p}{M} V_{z z z}(R)-R U_{z z z}(R)+U_{z z}(R)=0 .
\end{aligned}
$$

Put $\kappa=\left(\left(\nu_{j}^{2} \rho\right) /(E I)\right)^{1 / 4}, C=(E I) /\left(M \nu_{j}^{2}\right), \beta=U_{z z z}(R)$, and $\tilde{\beta}=U_{z z z}(R)$. Then (4.13) and the boundary conditions at $R$ yield

$$
\begin{aligned}
U(z+R)= & c_{1}(\cos (\kappa z)-\cosh (\kappa z))+c_{2}(\sin (\kappa z)-\sinh (\kappa z)) \\
& +C \beta(\cos (\kappa z)-1), \\
V(z+R)= & \tilde{c}_{1}(\cos (\kappa z)-\cosh (\kappa z))+\tilde{c}_{2}(\sin (\kappa z)-\sinh (\kappa z)) \\
& +C \tilde{\beta}(\cos (\kappa z)-1) .
\end{aligned}
$$

By definition of $\beta$ and $\tilde{\beta}$, we have

$$
\beta=-2 c_{2} \kappa^{3}, \quad \tilde{\beta}=-2 \tilde{c}_{2} \kappa^{3} .
$$

The free end boundary conditions are rewritten as

$$
\begin{aligned}
-c_{1}(\cos (\kappa L)+\cosh (\kappa L))-c_{2}(\sin (\kappa L)+\sinh (\kappa L))-C \beta \cos (\kappa L) & =0, \\
c_{1}(\sin (\kappa L)+\sinh (\kappa L))+c_{2}(\cos (\kappa L)+\cosh (\kappa L))-C \beta \sin (\kappa L) & =0,
\end{aligned}
$$

and precisely the same equations hold for $\tilde{c}_{1}, \tilde{c}_{2}$, and $\tilde{\beta}$. Solving these equations we obtain

$$
\begin{aligned}
2 c_{1}+C \beta & =-\frac{\sinh (\kappa L) \sin (\kappa L)}{1+\cosh (\kappa L) \cos (\kappa L)} C \beta, \\
2 c_{2} & =\frac{\cosh (\kappa L) \sin (\kappa L)+\sinh (\kappa L) \cos (\kappa L)}{1+\cosh (\kappa L) \cos (\kappa L)} C \beta, \\
2 \tilde{c}_{1}+C \tilde{\beta} & =-\frac{\sinh (\kappa L) \sin (\kappa L)}{1+\cosh (\kappa L) \cos (\kappa L)} C \tilde{\beta}, \\
2 \tilde{c}_{2} & =\frac{\cosh (\kappa L) \sin (\kappa L)+\sinh (\kappa L) \cos (\kappa L)}{1+\cosh (\kappa L) \cos (\kappa L)} C \tilde{\beta} .
\end{aligned}
$$


A comparison with (4.15) shows that either $\beta$ and $\tilde{\beta}$ are both 0 or

$$
\frac{\cosh (\kappa L) \sin (\kappa L)+\sinh (\kappa L) \cos (\kappa L)}{1+\cosh (\kappa L) \cos (\kappa L)}<0 .
$$

Since $\beta=0$ and $\tilde{\beta}=0$ would imply $c_{1}=c_{2}=\tilde{c}_{1}=\tilde{c}_{2}=0$, so that $\mathbf{u}=\mathbf{v}=0$, we infer that (4.17) holds. We notice also that, by (4.16), $\tilde{\beta} c_{1}=\beta \tilde{c}_{1}$ and $\tilde{\beta} c_{2}=\beta \tilde{c}_{2}$. Now we rewrite (4.14) as

$$
\begin{gathered}
\frac{2 M_{T} p}{M} \kappa^{3} c_{2}+2 R \kappa^{3} \tilde{c}_{2}-2 \kappa^{2} \tilde{c}_{1}-C \kappa^{2} \tilde{\beta}=0, \\
-\frac{2 M_{T} p}{M} \kappa^{3} \tilde{c}_{2}+2 R \kappa^{3} c_{2}-2 \kappa^{2} c_{1}-C \kappa^{2} \tilde{\beta}=0 .
\end{gathered}
$$

We multiply the first equation by $\beta$ and the second one by $\tilde{\beta}$ and add:

$$
4 R \kappa^{3} \beta \tilde{c}_{2}=\kappa^{2}\left(4 \beta \tilde{c}_{1}+2 C \beta \tilde{\beta}\right) .
$$

Dividing by $2 \kappa^{2} \beta \tilde{\beta}$ and utilizing (4.16) obtains

$$
2 R \kappa \frac{\cosh (\kappa L) \sin (\kappa L)+\sinh (\kappa L) \cos (\kappa L)}{1+\cosh (\kappa L) \cos (\kappa L)}=-\frac{\sinh (\kappa L) \sin (\kappa L)}{1+\cosh (\kappa L) \cos (\kappa L)} .
$$

Thus, by (4.17),

$$
\frac{\sinh (\kappa L) \sin (\kappa L)}{1+\cosh (\kappa L) \cos (\kappa L)}>0 .
$$

(4.17) and (4.18) together contradict Lemma 4.6. We have, therefore, shown that all $\mathbf{u}_{j}, \mathbf{v}_{j}$ vanish, i.e., $\dot{W}$ and $\phi$ are zero.

Proof of THE MAIN THEOREM . Since each trajectory of (3.1) is bounded, each sequence $t_{j} \rightarrow \infty$ contains a subsequence such that the corresponding states converge to a state in the weak $\omega$-limit set. Lemma 4.5 and Lemma 4.7 show that the states in the weak $\omega$-limit set are of the form $\theta=0, \dot{\theta}=0, \dot{W}=0, \phi=0$. From the conservation of momentum Lemma 3.3, we infer that $p$ and $q$ are now determined uniquely by the trajectory, so that the trajectory admits only one weak cluster point. Consequently, this is its weak limit. The weak limit 
for $W$ can be obtained from the boundary conditions $W(R)=R \theta$, $W_{z}(R)=\dot{\theta}$, and that $I W_{z z}(t, z)=\phi(t, 0, z)$.

\section{REFERENCES}

1. J. M. Ball, Strongly continuous semigroups, weak solutions and the variation of constants formula, Proc. Amer. Math. Soc. 63 (1977), 370-373.

2. - On the asymptotic behavior of generalized processes, with applications to nonlinear evolution equations, J. Differential Equations 27 (1978), 224-265.

3. W. Desch and R. K. Miller, Exponential stabilization of Volterra integrodifferential equations in Hilbert space, J. Differential Equations 70 (1987), 366-389.

4. - and - Exponential stabilization of Volterra integral equations with singular kernels, J. Integral Equations Appl. 1 (1988), 397-433.

5. J. Goldstein, Semigroups of linear operators and applications, Oxford University Press, New York, 1985.

6. J. Hale, Asymptotic behavior of dissipative systems, Math. Surveys and Monographs 25, Amer. Math. Soc., Providence, 1988.

7. A. Pazy, Semigroups of linear operators and applications to partial differential equations, Springer, New York, Berlin, 1983.

8. S. Skaar, Closed form optimal control solutions for continuous linear elastic systems, J. Astronautical Sciences 32 (1984), 447-461.

Department of Mathematics, Iowa State University, Ames, Iowa 50011, USA

Universität Graz, Institute für Mathematik, A-8010 Graz, Austria

Department of Aerospace and Mechanical Engineering, Notre Dame University, Notre Dame, Indiana 46556, USA 